\title{
Neuroimaging the Epileptogenic Process
}

\author{
Sandy R. Shultz • Terence J. O'Brien • \\ Maria Stefanidou • Ruben I. Kuzniecky
}

Published online: 1 February 2014

(C) The American Society for Experimental NeuroTherapeutics, Inc. 2014

\begin{abstract}
Epilepsy is one of the most common chronic neurological conditions worldwide. Anti-epileptic drugs (AEDs) can suppress seizures, but do not affect the underlying epileptic state, and many epilepsy patients are unable to attain seizure control with AEDs. To cure or prevent epilepsy, disease-modifying interventions that inhibit or reverse the disease process of epileptogenesis must be developed. A major limitation in the development and implementation of such an intervention is the current poor understanding, and the lack of reliable biomarkers, of the epileptogenic process. Neuroimaging represents a non-invasive medical and research tool with the ability to identify early pathophysiological changes involved in epileptogenesis, monitor disease progression, and assess the effectiveness of possible therapies. Here we will provide an overview of studies conducted in animal models and in patients with epilepsy that have utilized various neuroimaging modalities to investigate epileptogenesis.
\end{abstract}

Key Words Magnetic resonance imaging · Magnetic resonance spectroscopy $\cdot$ Diffusion weighted imaging · Positron emission tomography $\cdot$ Functional magnetic resonance imaging $\cdot$ Biomarkers $\cdot$ Epilepsy $\cdot$ Epileptogenesis

S. R. Shultz $(\bowtie) \cdot$ T. J. O’Brien

Department of Medicine, The Melbourne Brain Centre, The Royal Melbourne Hospital, The University of Melbourne, Building 144, Royal Parade, Parkville, VIC 3010, Australia

e-mail: sshultz@unimelb.edu.au

M. Stefanidou • R. I. Kuzniecky

Department of Neurology, Comprehensive Epilepsy Center, NYU Langone Medical Center, New York, NY, USA

\section{Introduction}

Epilepsy is a common neurologic disease characterized by the occurrence of spontaneous recurrent seizures [1]. Epilepsy affects approximately 50 million people worldwide [2], and holds an annual economic cost of over $\$ 12.5$ billion in the USA alone [3]. Although some epilepsy patients may be responsive to pharmaceutical treatments, at least $30 \%$ of patients are drug-resistant [4]. Furthermore, anti-epileptic drugs (AEDs) often have adverse side-effects and, even in drug-responsive cases, AEDs merely suppress seizures without stopping or interfering with the epileptogenic disease process that converts a healthy brain into an epileptic brain $[4,5]$. Therefore, therapies capable of preventing or reversing epileptogenesis are needed to cure this disease. Unfortunately, a major limitation in the development and implementation of such interventions is the poor understanding, and consequent lack of reliable biomarkers, of the epileptogenic process $[5,6]$.

Neuroimaging provides non-invasive research and medical tools with the capacity to identify early biomarkers involved in epilepsy, longitudinally monitor disease progression, and assess the effectiveness of epileptogenic therapies [7]. This article will review the research conducted at both the basic experimental and clinical level that has utilized various neuroimaging modalities to study epileptogenesis in vivo. The first section ('Neuroimaging Biomarkers in Animal Studies of Epilepsy') will present and evaluate studies that have utilized neuroimaging within animal models of epilepsy to identify potential biomarkers of epileptogenesis that may be translatable to the clinical setting. The second section ('Neuroimaging Biomarkers in Clinical Studies') will review the literature using neuroimaging in the clinical epilepsy setting. Detailed technical descriptions of the neuroimaging techniques and animal models that will be discussed are beyond the scope of this review, and readers are directed to the references provided in the appropriate sections for further information. 


\section{Neuroimaging Biomarkers in Animal Models of Epilepsy}

It is challenging to study early epileptogenic processes in the clinical setting as epilepsy is diagnosed in patients after the onset of spontaneous recurrent seizures, which represents the culmination of epileptogenesis [7]. Animal models of epilepsy, both acquired and genetic, provide the opportunity to rigorously investigate experimental subjects known to be experiencing epileptogenic processes, and thereby hold the potential to increase our understanding and identify early biomarkers of this disease. Briefly, some of the most commonly used and well-validated animal models of epilepsy that will be discussed in this section include exposure to proconvulsant chemicals, such as pilocarpine or kainic acid, to induce status epilepticus (SE) and consequent spontaneous seizures modeling temporal lobe epilepsy (TLE) [8]; electrical stimulation, or kindling, of temporal brain regions (e.g., amygdala) to model TLE [8]; and the fluid percussion brain injury (FPI) model of traumatic brain injury (TBI) and posttraumatic epilepsy (PTE) [9-11]. This section will provide a summary of the epileptogenic-related changes and neuroimaging biomarkers that have been identified to date within these models.

Magnetic Resonance Imaging: Signal, Volumetrics, and Contrast Agents

Magnetic resonance imaging (MRI) is based on the magnetic excitation of hydrogen nuclei in body tissue, and the consequent recording of the electromagnetic signals that are returned from the body [12]. Given its widespread clinical availability, non-invasive nature, and relative low cost, MRI is an attractive technique in both basic and clinical research. Specific to the basic experimental setting, epilepsy animal model studies have incorporated conventional MRI techniques for over 20 years [13], with $\mathrm{T}_{1}, \mathrm{~T}_{2}$, and $\mathrm{T}_{2}$-weighted (T2W) signal measures amongst the most commonly employed [14].

\section{Signal Changes}

$\mathrm{T}_{2}$ signal changes on MRI have been consistently reported at various phases of the epileptogenic process in numerous animal models, with the common underlying pathological changes being edema, gliosis, and cell loss (see Table 1) [15-18]. In chemoconvulsant SE models, increased $\mathrm{T}_{2}$ values and $\mathrm{T} 2 \mathrm{~W}$ hyperintensity have been reported in several brain regions, including the cortex, hippocampus, thalamus, amygdala [19, 20], and piriform and entorhinal cortices [15], beginning as early as $2 \mathrm{~h}$ after SE [20]. These acute changes typically return to baseline within $48-72 \mathrm{~h}[15,20]$, and have been reported to predict the development of epilepsy at 4 months after SE [15]. However, in contrast to these findings, other studies have reported a global decrease in $T_{2}$ values in the acute stages after SE [17], and evidence of T2W hyperintensity for up to 9 weeks after SE [16]. Consistent with chemically-induced SE models, SE induced by electrical stimulation of the rat amygdala resulted in increased $\mathrm{T}_{2}$ values in the amygdala beginning 2 days after SE [21], and increased T2W signal intensity in the hippocampus 2 weeks after kindling had ceased [22]. Taken together, these findings suggest temporal complexities in $\mathrm{T}_{2}$ signal changes occurring in post-SE TLE models, likely representing the evolving pathophysiological processes in epileptogenesis $[16,17]$ that must be clarified and considered when translating the use of $\mathrm{T}_{2}$-based biomarkers to the clinical setting.

Studies using animal models of PTE and febrile seizures (FS) have also reported $T_{2}$ signal changes possibly related to

Table 1 Neuroimaging biomarkers in animal models

\begin{tabular}{|c|c|c|c|}
\hline $\begin{array}{l}\text { Imaging } \\
\text { modality }\end{array}$ & Animal model & Potential biomarker & Related pathophysiology \\
\hline MRI-T2 signal & Post-SE, FPI, kindling, FS & Acute $\mathrm{T} 2$ signal increase and $\mathrm{T} 2 \mathrm{~W}$ hyperintensity & Edema, gliosis, cell loss \\
\hline MRI_volumetrics & Post-SE, FPI & Decreased volume of limbic structures (i.e., hippocampus) & Structural atrophy \\
\hline $\begin{array}{l}\text { MRI—contrast } \\
\text { agents }\end{array}$ & Post-SE & $\mathrm{Mn}^{2+}$ - and $\mathrm{Gd}^{3+}$-enhanced signal change & Mossy fibers and BBB breakdown \\
\hline DWI & $\begin{array}{l}\text { Post-SE, FPI, absence } \\
\text { epilepsy, FS }\end{array}$ & Altered ADC, FA, and tractography & Edema, axonal injury, connectivity \\
\hline MRS & Post-SE & $\begin{array}{l}\text { Reductions in glutamate, glutamine, GABA, and NAA, } \\
\text { and increased myo-inositol and glutathione }\end{array}$ & $\begin{array}{r}\text { Neurotransmitters, neuronal death } \\
\text { and dysfunction, glia activation }\end{array}$ \\
\hline PET & Post-SE, FPI & $\begin{array}{l}\text { Decreases in [18F]FDG-PET signal, increases in } \\
\text { [18F]PBR111-PET signal }\end{array}$ & Hypometabolism, inflammation \\
\hline fMRI & Post-SE, absence epilepsy & Changes in BOLD signal & Neuronal activity and metabolism \\
\hline
\end{tabular}

MRI = magnetic resonance imaging; DWI = diffusion-weighted MRI; MRS = magnetic resonance spectroscopy; PET = positron emission tomography; fMRI = functional MRI; $\mathrm{SE}=$ status epilepticus; $\mathrm{FPI}=$ fluid percussion brain injury; $\mathrm{FS}=$ febrile seizure; $\mathrm{T}_{2} \mathrm{~W}=\mathrm{T}_{2}$ weighted; $\mathrm{ADC}=$ apparent diffusion coefficient; FA = Fractional anisotropy; GABA = gamma-aminobutyric acid; NAA = N-acetyl aspartate; $\mathrm{BOLD}=$ blood oxygen level-dependent; $\mathrm{BBB}=$ blood-brain barrier 
epileptogenesis. In the FPI model of PTE, early increases in $\mathrm{T}_{2}$ signal values were found to be associated with functional and histological outcomes at both acute and chronic time-points [23]. However, it should be noted that these initial $\mathrm{T}_{2}$ changes were found to be poor predictors of subsequent decreases in seizure threshold [23]. In a hyperthermia-induced FS model, rats that experienced prolonged febrile seizures displayed increased T2W signal intensity at 1 and 8 days after FS [24], increased $\mathrm{T}_{2}$ values at 1 month after FS [25], and had an increased likelihood of developing spontaneous seizures after FS [24, 25]. However, the increase in $\mathrm{T}_{2}$ value itself was not found to be an accurate predictor of epileptogenesis [25]. Taken together, further research is required to determine the usefulness of $\mathrm{T}_{2}$-based biomarkers to predict the development of epileptogenesis that ultimately results in spontaneous recurrent seizures in the context of these models.

\section{Volumetric and Morphological Analyses}

In addition to signal changes, conventional $T_{1}$ and $T_{2}$ MRI also allow for volumetric and morphological analyses of brain structures involved in epileptogenesis. As shown in Fig. 1, volumetric and morphological changes in the hippocampus and other limbic structures have been reported in both SE
[26-29] and FPI models [11, 23, 30, 31]. However, these changes are typically preceded by abnormalities identified by other more sensitive neuroimaging methods [11, 23, 28-31]. As such, volumetric and morphological changes may not be ideal neuroimaging biomarkers to identify early pathophysiological processes involved in epileptogenesis, but may have more of a role to assess subsequent progressive neurodegenerative changes, in particular atrophy of key structures such as the hippocampus, that may follow an epileptogenic brain insult [11, 29, 30, 32].

\section{Contrast Agents}

The contrast in conventional MRI signal can be further enhanced by administration of exogenous contrast agents, such as manganese $\left(\mathrm{Mn}^{2+}\right)$ and gadolinium $\left(\mathrm{Gd}^{3+}\right)$ complexes. These agents have been used to study epileptogenesis in post-SE [16, 33-36] and FPI [32, 37] rodent models, and enable the assessment of various pathologies potentially involved in the epileptogenic process, such as blood-brain barrier breakdown [16, 37], mossy fiber sprouting [35], and reorganization of neural connections [31, 32]. For example, studies utilizing serial $\mathrm{Mn}^{2+}$-enhanced imaging at 2 days and 6 weeks after $\mathrm{SE}$ found increased $\mathrm{T}_{1}$-weighted signal intensity

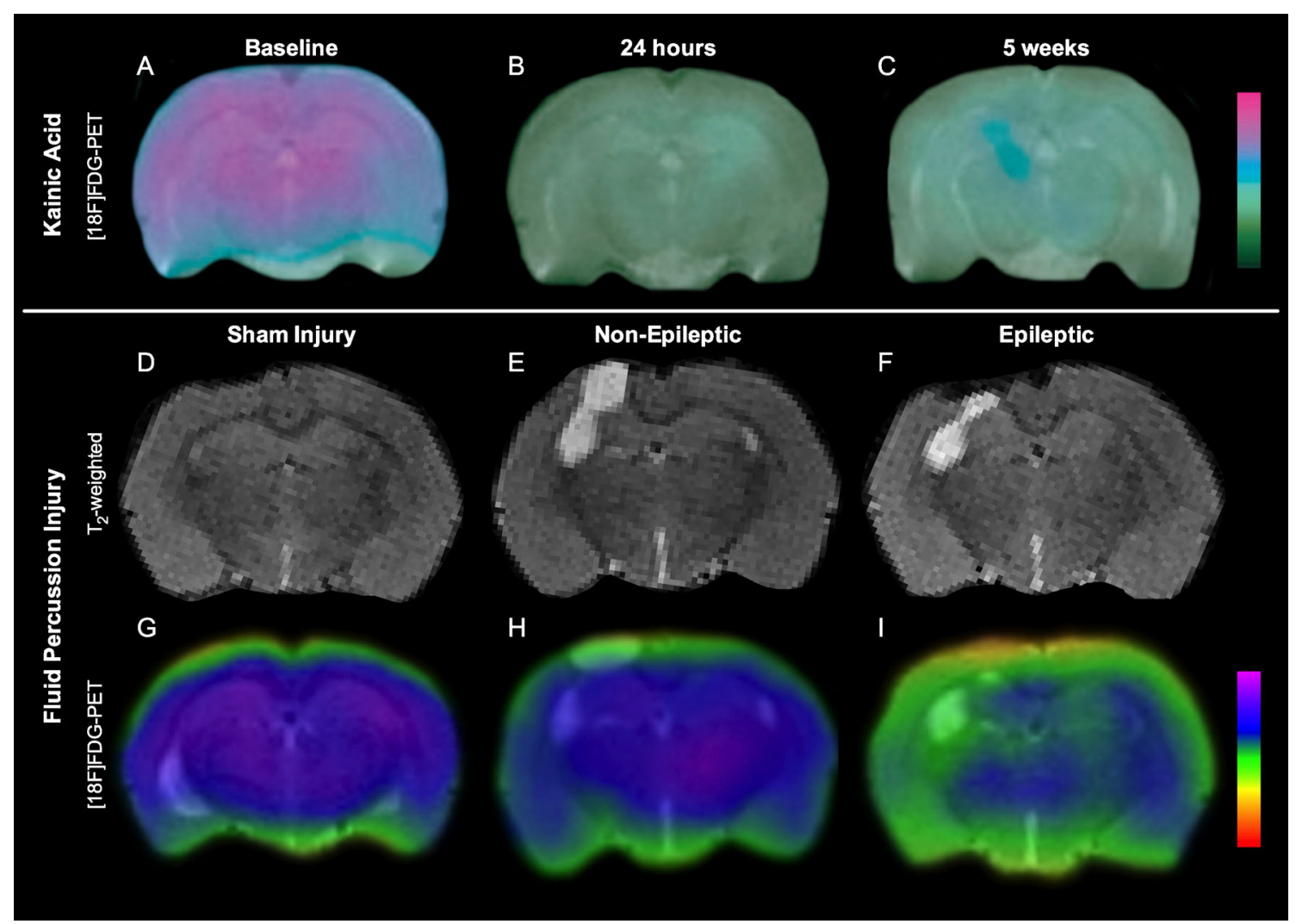

Fig. 1 Volumetric and [18F]FDG-positron emission tomography (PET) changes in kainic acid model of temporal lobe epilepsy and fluid percussion injury model of traumatic brain injury (TBI). There is persistent hypometabolism, and progressive degeneration after kainic acid-induced status epilepticus (A-C; adapted from [29]). Although all rats suffer progressive neurodegeneration after fluid percussion injury (D-F), rats that develop post-traumatic epilepsy display persistent hypometabolism at 3 months after TBI (G-I; adapted from [11]) 
in the hippocampus that were related to spontaneous seizure outcome in the chronic epilepsy phase, suggesting that $\mathrm{Mn}^{2+}$ enhanced imaging could provide a preclinical biomarker for the severity of epileptogenesis [34]. Unfortunately, a major limitation and confounder with the use of contrast agents, especially $\mathrm{Mn}^{2+}$, is their high toxicity in mammals $[14,30]$. However, these issues continue to be improved and, as such, contrast agents remain an effective tool in the study of epileptogenesis, particularly in the basic experimental setting.

\section{Diffusion-weighted MRI}

Diffusion-weighted MRI (DWI) and related methods, such as diffusion tensor and tractography analyses, are based on the quantification using magnetic resonance of the diffusivity of water molecules in the brain, which is more directionally limited (anisotropic) in white matter tracts relative to gray matter [36-38]. Common measures generated from DWI include apparent diffusion coefficient (ADC) - the magnitude of overall water diffusion in a given direction; mean diffusivity - the mean diffusivity across a number of directions; and fractional anisotropy (FA) - a value between 0 (isotropic) and 1 (anisotropic) that describes the anisotropy of diffusion [36]. In unhealthy white matter, damaged axonal membranes become less constricting to molecules and are thus less anisotropic [36, 37]. Tractography is another DWI-based postprocessing method that allows for the assessment of structural connectivity, and holds promise in the study of epileptogenesis [38]. Notably, changes in DWI-based measures are also commonly associated with edema [37].

In post-SE rat models of TLE an acute increase in ADC, beginning as early as 3 mins post-SE [39], has been reported $[17,40]$. However, this is a dynamic and structurallydependent response, with reports of acute decreases in ADC post-SE [17, 39-42], a return to baseline by 1 week, and increased ADC at chronic time-points [39]. ADC increases may be related to vasogenic edema or increased metabolic activity [17, 39, 43, 44], whereas the acute ADC decreases are associated with cytotoxic edema and neuronal loss [39, 43, 44]. Structural dependent changes in FA have also been identified at chronic epileptic stages after SE [45, 46]. Although chronic FA changes that occur after the manifestation of spontaneous seizures hold little value as an early biomarker for epileptogenesis, FA may be an effective biomarker if validated at earlier stages.

Changes in DWI outcomes have also been reported in the FPI [23, 31, 37] rat model of PTE at various stages in the epileptogenic process. Of particular importance, acute and chronic hippocampal ADC changes in the FPI model were associated with electroencephalography (EEG) parameters (i.e., total number of interictal epileptiform spikes and epileptiform discharges), mossy fibre sprouting, and seizure susceptibility after a chemoconvulsant challenge [23, 37]. It should also be noted that reductions in FA and associated tractography have been reported in animal models of genetic generalized epilepsy with absence seizures (i.e., WAG/Rij rats) [47], and decreased ADC and increased FA have been reported in rats after FS [48]. Taken together, DWI-based approaches are sensitive and promising in identifying potential epileptogenic-related changes throughout the disease process. However, future research is still required to assess whether these DWI changes are reliable biomarkers that predict epileptic outcomes [14].

\section{Magnetic Resonance Spectroscopy}

Magnetic resonance spectroscopy (MRS) is a neuroimaging technique capable of identifying levels of brain metabolites, such as N-acetyl aspartate (NAA), choline, creatine (Cr), lactate, myo-inositol, and glutathione; levels of neurotransmitters, such as glutamate, glutamine, and gamma-aminobutyric acid (GABA); and other physiological changes, such as intracerebral $\mathrm{pH}$, all of which may have relevance to epileptogenesis $[49,50]$. In post-SE animal models of TLE, MRS has identified acute reductions in glutamate, glutamine, and GABA [51,52], as well as acute and chronic reductions in NAA [50, 53-57], a marker of neuronal loss or dysfunction [49]. Furthermore, progressive increases in myo-inositol and glutathione have also been detected in acute stages after SE, and are maintained in epileptic rats [50]. Studies using the FPI model have also used MRS to identify acute abnormalities after injury $[58,59]$, though whether these changes are associated with PTE has yet to be determined. Abnormalities in NAA and Cr have also been reported after FS in rats and may be related to the later development of spontaneous seizures [48]. Overall, these findings demonstrate that MRS is capable of identifying biomarkers associated with numerous pathophysiological changes occurring throughout the epileptogenic process, and support its use in future investigations.

\section{Positron Emission Tomography}

Positron emission tomography (PET) uses radiotracers to visualize and quantify functional processes in the brain. A variety of radiotracers are available, including those capable of identifying pathophysiological processes recently implicated in epileptogenesis, such as metabolism, neuroinflammation, and hyperphosphorylated tau [60-64]. For example, in vivo [18F]PBR111-PET has found significant inflammation after SE in the hippocampus and amygdala during epileptogenesis [60]. To date, the most common radiotracer employed in animal epilepsy studies, as in human epilepsy practice and research, is the glucose analogue [18F]FDGPET, a biomarker for glucose uptake and brain metabolism. As shown in Fig. 1, in chemoconvulsant post-SE rat models, [18F]FDG-PET signal has been consistently found to 
decrease during the acute post-SE period $[29,57,65,66]$, with some studies reporting a return to baseline at the subacute stage followed by decreased metabolism again in the chronic epileptic stage [29, 57]. Importantly, it has been reported that hypometabolism precedes, and is unrelated to, the atrophy of limbic structures, suggesting that the hypometabolism is not simply an effect of atrophy and may indicate cellular changes important in epileptogenesis [29]. A significant relationship has been reported between the degree of hypometabolism on [18F]FDG-PET in the entorhinal cortex early following lithium pilocarpine-induced $\mathrm{SE}$ in the rat and the chance of later developing spontaneous recurrent seizures, suggesting that this may be a biomarker of early epileptogenesis following a brain insult [66]. As seen in Fig. 1, [18F]FDG-PET following FPI in rats has also identified acute and persistent hypometabolism that preceded significant structural atrophy $[11,30]$, and may predict the later onset of PTE [11]. While further research is clearly required, and other limitations must be considered [62], these findings, together with the ability to assess other relevant pathophysiological processes in vivo, make PET a valuable experimental tool in the investigation of epileptogenesis.

\section{Functional MRI}

As with PET, functional MRI (fMRI) allows for the assessment of functional brain activity. However, fMRI is less expensive, less invasive, and provides superior temporal and spatial resolution relative to other functional neuroimaging techniques. For example, fMRI has temporal and spatial resolutions in the order of hundreds of milliseconds and one millimeter [12, 67, 68], whereas PET has temporal and spatial resolutions in the order of minutes and millimeters [62, 68]. Considering these factors, fMRI is now the most commonly used functional imaging method [12, 67]. Ogawa et al. [69] discovered the basic principle of fMRI, that MRI signal is sensitive to changes in blood oxygenation levels, over 20 years ago. As neuronal activity leads to increased blood flow and changes in blood oxygenation levels, or the blood oxygenation level-dependent (BOLD) signal, fMRI allows for the investigation of the location and networks of brain function $[12,67]$. As epilepsy is known to involve abnormalities in neural activity and circuitry, fMRI represents a promising tool in the study of epileptogenesis [67].

Animal models of epilepsy are advantageous for fMRI studies as they allow for control over the type and timing of seizure, elimination of movement artifacts, and the use of more invasive techniques to complement fMRI analysis [67]. Studies using chemoconvulsant SE models have reported robust BOLD changes associated with seizure activity [67, 70-72]. Similarly, fMRI studies in animal models of genetic generalized epilepsy with absence epilepsy also report BOLD signal abnormalities associated with spontaneous spike-wake discharges [67, 73-75]. Though there have been some inconsistencies regarding these findings (i.e., BOLD increase vs decrease) [76], and other potential confounds and limitations (e.g., animals are often under general anesthetic) to consider [67], these studies clearly demonstrate the ability and usefulness of fMRI in identifying functional abnormalities in epileptogenesis.

\section{Neuroimaging Biomarkers in Clinical Studies}

Many different neuroimaging modalities are utilized in the evaluation of patients with epilepsy. It is more challenging, though, to track the epileptogenic process in the clinical setting as the majority of our data come from patients with chronic epilepsy and the changes seen on imaging may reflect, to varying degrees, the genetics, the pathogenic process, aging, secondary damage, or even drug effects [77].

Ideally, a biomarker for epileptogenesis would help diagnose and predict epilepsy in the preclinical or early symptomatic stage, it would be specific to the epileptic process, it would monitor disease progression, and demonstrate reversal of epileptogenesis following therapeutic interventions [78, 79].

In order to help elucidate the role of imaging in the development of the epileptogenic process in humans, we will focus in this portion of the review on data from longitudinal clinical studies that may infer causality or help document disease progression. Clinical settings where neuroimaging has been used to study epileptogenesis include FS, PTE, early-onset epilepsy in children, and studies assessing long-term disease prognosis in chronic epilepsy.

\section{MRI}

\section{Signal Changes}

There has long been observed an association between FS and development of TLE later in life. In children, immediately after complex FS or prolonged status, increased $\mathrm{T}_{2} / \mathrm{DWI}$ signal has been reported in the hippocampus [80, 81], ipsilateral thalamus, and anterior cingulate gyrus [82]. These acute changes are reversible [83] and thought to be secondary to cytotoxic or vasogenic edema. Concurrent EEG background abnormalities and focal spikes have also been reported [82]. Increased $\mathrm{T}_{2}$ signal and hippocampal volume changes, in this setting, are the most predictive indicators of subsequent development of mesial temporal sclerosis (MTS) [7, 81, 84].

In the FEBSTAT study, a longitudinal study to try and elucidate the pathogenic link between FS and late development of epilepsy, $11.5 \%$ of children presenting with prolonged ( $>30 \mathrm{~min}$ ) febrile status had increased or equivocal $\mathrm{T}_{2}$ signal in the hippocampus and more widespread in the 
temporal lobe, compared with none among children with simple febrile seizures [85]. T2W hippocampal signal abnormality was associated with focal EEG slowing or attenuation suggestive of acute hippocampal injury [86].

In TBI, the presence of cortical or subcortical $\mathrm{T}_{2}$ hyperintense lesions 1 year after the initial insult is associated with an increased risk of PTE [87]. Furthermore, on $\mathrm{T}_{1}$-weighted magnetization transfer images, abnormalities extending beyond the $T_{2}$ changes or presence of gliosis surrounding hemosiderin deposits (especially when the gliosis wall is incomplete or shows a dynamic evolution in subsequent scans) are associated with higher risk for epilepsy [88, 89]. Furthermore, hippocampal $\mathrm{T}_{2}$ increased signal and atrophy have been shown to be strong indicators of seizure recurrence following AED withdrawal in seizure-free patients [90].

The above findings of mainly $T_{2}$ signal abnormalities seen on structural MRI are suggestive of a potentially epileptogenic effect of both acute and chronic neural injury, but the causal link is still under investigation. In the case of FS, the long-term outcomes of the FEBSTAT study will hopefully shed more light into the significance of acute signal changes and their link to epileptogenesis.

\section{Volumetric and Morphological Analysis}

Some of the morphometric findings seen on MRI raise the possibility of a direct contribution to epileptogenesis. In the FEBSTAT study, developmental abnormalities of the hippocampus (hippocampal malrotation being the most common) were seen in the group of children with prolonged FS, suggestive of a possible predisposition to the initial epileptogenic insult [85]. Seizure-generating hypothalamic hamartomas appear to always have a connection to one or both mammillary bodies [91].

Cortical dysgenesis and development of subsequent epilepsy is well known, but prognosis after surgical removal of the visible structural abnormality varies. Lack of gray or white matter volume distribution abnormalities outside the primary lesion in the preoperative volumetric MRI is associated with better prognosis following lesionectomy [92]. Presence on MRI of thickened gyri and focal versus hemispheric involvement with severe hypometabolism on [18F]FDG-PET is a better predictor of specific epileptogenic cells than interictal or ictal EEG, and therefore may be used as a biomarker of epileptogenic tissue [93].

Gray matter volume reduction also correlates with epilepsy duration and has been reported for the hippocampus, entorhinal cortex [94], and thalamus [7]. It is more widespread in refractory TLE [90] and more severe in patients with MTS [7]. In TLE, MR volumetry has demonstrated bilateral white [95] and gray matter changes, and a history of FS is associated with smaller thalamic volumes ipsilateral to the seizure focus [82].
In familial mesial TLE, progression of hippocampal atrophy is seen in patients with frequent seizures and poor outcome [96].

Neocortical atrophy can be quantified by measuring cortical thickness across the entire cortical mantle to track continuous changes [97]. Cross-sectional analysis in refractory TLE showed progressive atrophy in the ipsilateral orbitofrontal, mesiotemporal, postcentral, and contralateral prefrontal areas. Longitudinal analysis over 2.5 years showed ipsilateral temporopolar and central, as well as contralateral orbitofrontal, insular, and angular region, cortical atrophy. Atrophy in somatomotor and parahippocampal regions in medial temporal lobe epilepsy (MTLE) has also been reported [94]. In patients with idiopathic generalised epilepsy (IGE), a 3.2 \% decrease in global mean cortical thickness and focal cortical thinning in frontal, precentral, central, and lateral temporal regions has been described. Increased seizure frequency showed faster cortical thinning [97]. Contrary to the above findings, larger septal nuclei are reported in patients with TLE without MTS compared with extratemporal epilepsy, and controls raise a possible prophylactic effect against epileptogenesis of these structures [98].

Taken together, the above findings show that morphological change may be a direct marker of intrinsic epileptogenesis, but - as in animal models-MRI volumetric analysis plays more of a role in describing disease progression. Higher extracellular glutamate concentration (a highly excitotoxic molecule) has been associated with decreased ipsilateral epileptogenic hippocampal volume and history of increased frequency of seizures [99], but a causal link to epileptogenesis has not yet been demonstrated. Unfortunately, most longitudinal MRI epilepsy studies have had a small number of patients or limited follow-up. Therefore, long-term longitudinal documentation of these changes may help characterize the epileptogenic process per se in the future [94].

\section{DWI}

White matter is an integral part of the epileptic network and assessment of its integrity, as discussed in the animal studies, may offer further insight in understanding the epileptogenic process. DWI changes are thought to be an effect of ongoing neuronal damage representing downstream axonal degeneration. Correlation between duration of epilepsy and DWI abnormalities has been shown in some white matter tracts, especially in TLE without MTS [95]. In children with TLE there are reports of decreased FA in the hippocampi ipsilateral and contralateral to the seizure focus [7], in the uncinate fasciculus, arcuate fasciculus, internal longitudinal fasciculus, and cortical spinal tract, as well as increased mean diffusivity, but normal FA in the temporal lobe white matter and cingulate gyrus [95]. In patients with TLE there is reduced FA in the external capsule and corpus callosum [95]. When unilateral MTS is present, DWI shows more extensive, bilateral 
decrease in white matter integrity [77] and bilateral fornix abnormalities [100].

In frontal cortical dysplasia (FCD) among patients with and without epilepsy, DWI abnormalities extend beyond the visible FCD abnormalities in both groups, but when white matter tracts projecting to and from the FCD where evaluated, those with seizures had more severe changes [101].

Following a single event of SE there was progressive atrophy of the left hippocampus with abnormal mean diffusivity over a period of 6 months thought to be secondary to delayed programmed cell death [100].

In a group of 5 children with epilepsy and large cavum septum pellucidum, use of DWI identified that the fornix body was consistently split into 2 bundles; the authors postulated a possible link to epileptogenesis, given the close association of the fornix to the limbic system [102].

DWI studies may prove a useful method for recording secondary cerebral damage, and more studies are needed to document the reproducibility of results and their potential prognostic utility in chronic epilepsy.

\section{PET}

\section{$[18 F] F D G-P E T$}

Metabolic dysfunction of neurons and glia is common in epilepsy and often specific enough to identify the seizure onset zone. As a measure of total glucose consumption $[18 \mathrm{~F}] \mathrm{FDG}-\mathrm{PET}$ is commonly used to identify hypometabolic areas [103].

In a series of patients with Sturge-Weber syndrome, transient interictal hypermetabolism on [18F]FDG-PET was documented in the posterior or frontal lobes within a short time before or after the onset of the first clinical seizures thought to be secondary to chronic ischemia inducing cortical damage and excitotoxicity. These changes were then followed by progressive loss of metabolism as epilepsy established itself [104].

A longitudinal study of 170 patients looking into long-term prognosis after hemispherectomy reported that hypometabolic abnormalities on [18F]FDG-PET may be a more reliable marker of potential independent epileptogenesis in the contralateral hemisphere than the structural abnormalities seen on brain MRI [105].

\section{a-[11C]Methyl-L-tryptophan}

a-[11C]Methyl-L-tryptophan tracer has been successful in children with tuberous sclerosis in differentiating epileptic from non-epileptic tubers. This particular radiotracer is interesting, as preliminary data suggest that its predilection to epileptogenic tissue may be due to increased focal activity of inflammatory pathways. Another radiotracer that has been found in activated microglia is [11C]-(R)-PK11195. Given the fact that neuroinflammation plays a potential role in epileptogenesis, these tracers might be useful in tracking response to immunotherapy that is already available [106].

\section{5-HT1A}

Decreased 5-HT1A receptor binding correlated with the degree of epileptogenesis in regions involved in seizure onset and where discharges propagated. Reduced free fractioncorrected volume of distribution and significantly greater asymmetry was described in the fusiform gyrus, hippocampus, and parahippocampus ipsilateral to epileptic foci [7].

\section{${ }^{11}$-flumazenil}

Complex mechanisms of epileptogenesis are characterized by metabolic and neurotransmitter/receptor disturbances. Functional impairment of inhibitory neurotransmission is probably a major factor. ${ }^{11} \mathrm{C}$-flumazenil is a PET tracer that serves as a useful in vivo marker of central inhibitory GABA A-type benzodiazepine receptor. ${ }^{11} \mathrm{C}$-flumazenil decreases are largely congruent with seizure onset zone and more severely depressed in the epileptogenic lesion than in adjacent cortex. Reductions have been described with various etiologies of seizures, in resected hippocampi, in spiking cortex, and in perilesional epileptogenic cortex with a highly variable pattern [107].

\section{Single Photon Emission Computed Tomography}

Single photon emission computed tomography (SPECT) studies use tracers sensitive to cerebral blood flow. One such tracer of central GABA-A benzodiazepine receptor binding is ${ }^{123} \mathrm{I}$ iomazenil. Studies in mesial TLE showed decreased binding that correlated with decreased neuronal density in the hippocampus and dentate gyrus. Similar changes were present in neocortical dysplasia, even in the absence of structural MRI lesions or SPECT studies evaluating cerebral blood flow [108, 109]. Among patients who had severe TBI, hypoperfusion in the temporal lobes 1 year after trauma, as measured with $99 \mathrm{~m}$ Tc hexamethyl-propyleneamine-oxime, correlated highly with the development of PTE [110]. However, despite this finding, no studies to date have suggested a role for SPECT as a biomarker for epilepsy.

\section{MRS}

MRS as a marker of epileptogenesis has primarily focused on measurements of the NAA/Cr ratio, reduction of which has been linked to neuronal mitochondrial injury and neuronal dysfunction. 
In one study among patients with IGE, thalamic MRS imaging measuring $\mathrm{NAA} / \mathrm{Cr}$ showed progressive thalamic neuronal dysfunction without volume decrease supporting the notion of abnormal thalamo-cortical circuitry as a substrate of seizure generation. There is significant reduction in thalamic NAA/Cr compared with normal controls that correlates with disease duration, but not the age of onset [111].

An intimate relation between interictal epileptiform discharges and reduction of the NAA/Cr ratio in the contralateral side has been described in MTLE patients, and it may be a possible marker of epileptogenesis of the contralateral side [112]; it has been associated with worse outcomes following epilepsy surgery [113]. Along the same lines, MRS in patients with TLE who failed to respond to the first AED trial had significantly lower NAA/Cr ratios compared with responders, possibly reflecting the different nature of the underlying epileptogenesis [90]. In one case of TLE with unilateral MTS there was normalization of the $\mathrm{NAA} / \mathrm{Cr}$ ratio in the contralateral temporal lobe following successful temporal lobe resection [7], raising the possibility of a biomarker that could potentially track reversal of epileptogenesis.

Comparison of NAA/Cr data of 12 different limbic foci between resting controls and MTLE patients showed that although both groups shared a metabolic network between the thalami and hippocampi, in patients there was an additional metabolic covariance seen between the ipsilateral insula and basal ganglia. The authors postulated it may represent downstream metabolic injury linked to seizure spread, and it remains to be proven if this network plays a role in the late recurrence of seizures after epilepsy surgery [114].

\section{fMRI}

BOLD signal changes have helped identify epileptogenic networks that not only correlate with seizure foci, but which are also seen at sites distant from EEG foci [77]. Among MTLE patients, compared with resting controls, there is decreased basal functional connectivity seen in the epileptogenic medial temporal lobe [115-117]. Decreased functional connectivity has been described in left mesial TLE across a network that includes thalamic, brainstem, frontal, and parietal regions, as well as focally in the dorsal medial prefrontal cortex, mesial temporal lobe, and inferior temporal cortex compared with controls [7]. However, no conclusive studies have shown a direct correlation between the bold signal and seizure progression.

Taken together, PET, SPECT, MRS, and fMRI studies that detect cerebral function are proving to be more sensitive in identifying an "epileptic network" and offer a more dynamic look into the epileptogenic process, with some evidence also alluding to reversibility of findings following therapeutic interventions.

\section{Future Directions and Conclusions}

There is currently no therapy proven to prevent or reverse epileptogenesis in patients. To develop and implement such an intervention, the underlying disease mechanisms, and related biomarkers, must first be identified and validated. Therapies can then be discovered, implemented, and rigorously investigated in preclinical studies that make use of the epileptogenic biomarkers to monitor disease progression. If a biomarker is modified by therapeutic intervention, and this correlates with reversal of the epileptogenic process, these therapies and biomarkers may then be valuable as surrogate endpoints in well-designed clinical trials. As highlighted in this review, neuroimaging represents a non-invasive and clinically applicable technique that can contribute to each of these needs. Methods such as MRI, PET, MRS, fMRI, and DWI are capable of assessing, or have already been associated with, a number of pathophysiological changes occurring in epileptogenesis. Future preclinical and clinical studies that continue to identify and validate neuroimaging biomarkers, improve imaging methods, use multimodal imaging, and combine imaging with other validated techniques (e.g., EEG), will make further strides towards our better understanding of the epileptogenic process. There is also growing evidence from animal models that epilepsy is a preventable disorder (see [118] for a review), with the identification of promising antiepileptogenic treatments, including rapamycin and sodium selenate $[118,119]$. However, to maximize the translatability of these findings to human patients it is important that future preclinical anti-epileptogenic studies utilize valid and clinically applicable biomarkers, such as neuroimaging, in their assessment of these therapies [e.g., 120]. To date, the few human clinical trials investigating potential antiepileptogenesis treatments have failed to demonstrate significant efficacy (see [121] and [122] for reviews). However, these failures have occurred in trials using therapies that lacked preclinical evidence of anti-epileptogenic effects and/or had major methodological shortcomings [121, 122]. In light of these failures, future anti-epileptogenic clinical trials should select a patient population with a high epilepsy risk or early in the disease process, include large sample sizes, employ a longitudinal study design with long observation periods, implement a treatment with strong preclinical evidence of anti-epileptogenic effects, and incorporate biomarkers, such as neuroimaging, that are reproducible, reliable, safe, and widely available [121, 122]. Neuroimaging provides promising epieptogenic biomarkers that are likely to play an integral role in our quest to develop interventions that are effective at preventing or reversing epilepsy.

Acknowledgments The authors declare there is no real or perceived conflict of interest. Full conflict of interest disclosures are available in the electronic supplementary material for this article. 
Required Author Forms Disclosure forms provided by the authors are available with the online version of this article.

\section{References}

1. Commission on Classification and Terminology of the International League Against Epilepsy (ILAE). Guidelines for epidemiologic studies on epilepsy Epilepsia 1993;34:592-596.

2. Banerjee PN, Filippi D, Hauser WA. The descriptive epidemiology of epilepsy - a review. Epilepsy Res 2009;85:31-45.

3. Begley CE, Famulari M, Annegers JF, et al. The cost of epilepsy in the United States: an estimate from population-based clinical and survey data. Epilepsia 2000;41:342-351.

4. Kwan P, Schachter SC, Brodie MJ. Drug-resistant epilepsy. N Eng1 J Med 2011;365:919-926.

5. Loeb JA. Identifying targets for preventing epilepsy using systems biology. Neurosci Lett 2011;497:205-212.

6. Dudek FE, Staley KJ. The time course of acquired epilepsy: implications for therapeutic intervention to suppress epileptogenesis. Neurosci Lett 2011;497:240-246.

7. Mishra AM, Bai H, Gribizis A, Blumenfeld H. Neuroimaging biomarkers of epileptogenesis. Neurosci Lett 2011;497:194-204.

8. Löscher W. Animal models of epilepsy for the development of antiepileptogenic and disease-modifying drugs. A comparison of the pharmacology of kindling and post-status epilepticus models of temporal lobe epilepsy. Epilepsy Res 2002;50:105-123.

9. Thompson HJ, Lifshitz J, Marklund N, et al. Lateral fluid percussion brain injury: A 15-year review and evaluation. J Neurotrauma 2005; 22: 42-75.

10. Pitkänen A, Bolkvadze T, Immonen R. Anti-epileptogenesis in rodent post-traumatic epilepsy models. Neurosci Lett 2011;497: 163-171.

11. Shultz SR, Cardamone L, Liu YR, et al. Can structural or functional changes following traumatic brain injury in the rat predict epileptic outcome? Epilepsia 2013;54:1240-1250

12. Goebel R. Localization of brain activity using functional magnetic resonance imaging. In: Stippich $\mathrm{C}$ (ed.) Clinical functional MRI. Berlin, Springer, 2007, pp. 9-51.

13. Karlik SJ, Stavraky RT, Taylor AW, Fox AJ, McLachlan RS. Magnetic resonance imaging and 31P spectroscopy of an interictal cortical spike focus in the rat. Epilepsia 1991;32:446-453.

14. Nehlig A. Hippocampal MRI and other structural biomarkers: experimental approach to epileptogenesis. Biomark Med 2011:5:585-597.

15. Roch C, Leroy C, Nehlig A, Namer IJ. Predictive value of cortical injury for the development of temporal lobe epilepsy in 21-day-old rats: an MRI approach using the lithium-pilocarpine model. Epilepsia 2002;43:1129-1136.

16. Roch C, Leroy C, Nehlig A, Namer IJ. Magnetic resonance imaging in the study of the lithium-pilocarpine model of temporal lobe epilepsy in adult rats. Epilepsia 2002;43:325-335.

17. van Eijsden $\mathrm{P}$, Notenboom RG, Wu O, et al. In vivo $1 \mathrm{H}$ magnetic resonance spectroscopy, T2-weighted and diffusion-weighted MRI during lithium-pilocarpine-induced status epilepticus in the rat. Brain Res 2004;1030:11-18.

18. Hsu Y, Lee WT, Chang C. Multiparametric MRI evaluation of kainic acid-induced neuronal activation in rat hippocampus. Brain 2007;130:3124-3134.

19. Fabene PF, Marzola P, Sbarbati A, Bentivoglio M. Magnetic resonance imaging of changes elicited by status epilepticus in the rat brain: diffusion-weighted and $\mathrm{T} 2$-weighted images, regional blood volume maps, and direct correlation with tissue and cell damage. Neuroimage 2003;18:375-389.
20. Fabene PF, Weiczner R, Marzola P, et al. Structural and functional MRI following 4-aminopyridine-induced seizures: a comparative imaging and anatomical study. Neurobiol Dis 2006; 21:80-89.

21. Nairismägi J, Grohn OH, Kettunen MI, Nissinen J, Kauppinen RA, Pitkanen A. Progression of brain damage after status epilepticus and its association with epileptogenesis: a quantitative MRI study in a rat model of temporal lobe epilepsy. Epilepsia 2004; 45:1024-1034.

22. Jupp B, Williams JP, Tesiram YA, Vosmansky M, O’Brien TJ. Hippocampal T2 signal change during amygdala kindling epileptogenesis. Epilepsia 2006; 47:41-46.

23. Kharatishvili I, Immonen R, Grohn O, Pitkänen A. Quantitative diffusion MRI of hippocampus as a surrogate marker for posttraumatic epileptogenesis. Brain 2007;130:3155-3168.

24. Dube C, Yu H, Nalcioglu O, Baram TZ. Serial MRI after experimental febrile seizures: altered T2 signal without neuronal death. Ann Neurol 2004;56:709-714.

25. Dube CM, Ravizza T, Hamamura M, et al. Epileptogenesis provoked by prolonged experimental febrile seizures: mechanisms and biomarkers. J Neurosci 2010;30:7484-7494.

26. Wolf OT, Dyakin V, Patel A, et al. Volumetric structural magnetic resonance imaging (MRI) of the rat hippocampus following kainic acid (KA) treatment. Brain Res 2002;934:87-96.

27. Niessen HG, Angenstein F, Vielhaber S, et al. Volumetric magnetic resonance imaging of functionally relevant structural alterations in chronic epilepsy after pilocarpine-induced status epilepticus in rats. Epilepsia 2005;46:1021-1026.

28. Choy M, Cheung KK, Thomas DL, Gadian DG, Lythgoe MF, Scott RC. Quantitative MRI predicts status epilepticus-induced hippocampal injury in the lithium-pilocarpine rat model. Epilepsy Res 2010;88:221-230.

29. Jupp B, Williams J, Binns D, Hicks RJ, Cardamone L, Jones N, Rees S, O'Brien TJ. Hypometabolism precedes limbic atrophy and spontaneous recurrent seizures in a rat model of TLE. Epilepsia 2012;53:1233-1244.

30. Liu YR, Cardamone L, Hogan RE, et al. Progressive metabolic and structural cerebral perturbations after traumatic brain injury: an in vivo imaging study in the rat. J Nucl Med 2010;51:1788-1795

31. Immonen R, Kharatishvili I, Grohn O, Pitkanen A. MRI biomarkers for post-traumatic epileptogenesis. J Neurotrauma 2013;30:13051309.

32. Bouilleret V, Cardamone L, Liu YR, et al. Confounding neurodegenerative effects of manganese for in-vivo MR imaging in rat models of brain insults. J Magn Reson Imaging 2011;34:774-784.

33. Nairismägi J, Pitkänen A, Narkilahti S, Huttunen J, Kauppinen RA, Gröhn $\mathrm{OH}$. Manganese-enhanced magnetic resonance imaging of mossy fiber plasticity in vivo. Neuroimage 2006;30:130-135.

34. Immonen RJ, Kharatishvili I, Sierra A, Einula C, Pitkanen A, Grohn $\mathrm{OH}$. Manganese enhanced MRI detects mossy fiber sprouting rather than neurodegeneration, gliosis or seizure-activity in the epileptic rat hippocampus. Neuroimage 2008;40:1718-1730.

35. Malheiros JM, Polli RS, Paiva FF, et al. Manganese-enhanced magnetic resonance imaging detects mossy fiber sprouting in the pilocarpine model of epilepsy. Epilepsia 2012;53:1225-1232.

36. Dedeurwaerdere S, Fang K, Chow M, et al. Manganese-enhanced MRI reflects seizure outcome in a model of mesial temporal lobe epilepsy. Neuroimage 2013;68:30-38.

37. Frey L, Lepkin A, Schickedanz A, Huber K, Brown MS, Serkova N. ADC mapping and T1-weighted signal changes on post-injury MRI predict seizure susceptibility after experimental traumatic brain injury. Neurol Res 2014;36:26-37.

38. Jones DK. Studying connections in the living human brain with diffusion MRI. Cortex 2008:44:936-952.

39. Yagarajah M, Duncan JS. Diffusion-based magnetic resonance imaging and tractography in epilepsy. Epilepsia 2008;49:189-200.

40. Engel J, Thompson PM, Stern JM, Staba RJ, Bragin A, Mody I. Connectomics and epilepsy. Curr Opin Neurol 2013;26:186-194. 
41. Zhong J, Petroff OAC, Prichard JW, Gore JC. Changes in water diffusion and relaxation properties of rat cerebrum during status epilepticus. Magn Reson Med 1993;30:241-246.

42. Prichard JW, Zhong J, Petroff OA, Gore JC. Diffusion-weighted NMR imaging changes caused by electrical activation of the brain. NMR Biomed 1995;8:359-364.

43. Engelhorn T, Hufnagel A, Weise J, Baehr M, Doerfler A. Monitoring of acute generalized status epilepticus using multilocal diffusion MR imaging: early prediction of regional neuronal damage. AJNR Am J Neuroradiol 2008;28:321-327.

44. Wall CJ, Kendall EJ, Obenaus A. Rapid alterations in diffusionweighted images with anatomic correlates in a rodent model of status epilepticus. AJNR Am J Neuroradiol 2000;21:1841-1852.

45. Laitinen T, Sierra A, Pitkänen A, Gröhn O. Diffusion tensor MRI of axonal plasticity in the rat hippocampus. Neuroimage 2010;51:521530.

46. Sierra A, Laitinen T, Lehtimäki K, Rieppo L, Pitkänen A, Gröhn O. Diffusion tensor MRI with tract-based spatial statistics and histology reveals undiscovered lesioned areas in kainate model of epilepsy in rat. Brain Struct Funct 2011;216:123-135.

47. Chahboune H, Mishra AM, DeSalvo MN, et al. DTI abnormalities in anterior corpus callosum of rats with spike-wave epilepsy, Neuroimage 2009;47:459-466.

48. Jansen JFA, Lemmens EMP, Strijkers GJ, et al. Short- and long-term limbic abnormalities after experimental febrile seizures. Neurobiol Dis 2008;32:293-301.

49. Duncan JS. Magnetic resonance spectroscopy. Epilepsia 1996;37: 598-605.

50. Filibian M, Frasca A, Maggioni D, Micotti E, Vezzani A, Ravizza T. In vivo imaging of glia activation using $1 \mathrm{H}$-magnetic resonance spectroscopy to detect putative biomarkers of tissue epileptogenicity. Epilepsia 2012;53:1907-1916.

51. Zahr NM, Crawford EL, Hsu O. In vivo glutamate decline associated with kainic acid-induced status epilepticus. Brain Res 2009;1300:65-78.

52. Alvestad S, Hammer J, Qu H, Håberg A, Ottersen OP, Sonnewald U. Reduced astrocytic contribution to the turnover of glutamate, glutamine, and GABA characterizes the latent phase in the kainate model of temporal lobe epilepsy. J Cereb Blood Flow Metab 2011;31:1675-1686.

53. Najm IM, Wang Y, Hong SC, Luders HO, Ng TC, Comair YG. Temporal changes in proton MRS matabolites in rat brain. Epilepsia 1997;38:87-94.

54. Ebisu T, Rooney WD, Graham SH, Mancuso A, Weiner MW, Maudsley AA. MR spectroscopic imaging and diffusion-weighted MRI for early detection of kainite induced status epilepticus in the rat. Magn Reson Med 1996;36:821-828.

55. Gomes WA, Lado FA, De Lanerolle NC, Takahashi K, Pan C, Hetherington HP. Spectroscopic imaging of the pilocarpine model of human epilepsy suggests that early NAA reduction predicts epilepsy. Magn Reson Med 2007;58:230-235.

56. Tokumitsu T, Mancuso A, Weinstein PR, Weiner MW, Naruse S, Maudsley AA. Metabolic and pathological effects of temporal lobe epilepsy in rat brain detected by proton spectroscopy and imaging. Brain Res 1997;744:57-67.

57. Lee EM, Park GY, Im KC, et al. Changes in glucose metabolism and metabolites during the epileptogenic process in the lithiumpilocarpine model of epilepsy. Epilepsia 2012;53:860-869.

58. Kleindienst A, Tolias CM, Corwin FD, et al. Assessment of cerebral S100B levels by proton magnetic resonance spectroscopy after lateral fluid-percussion injury in the rat. J Neurosurg 2005;102:1115-1121.

59. Vink R, Faden AI, McIntosh TK. Changes in cellular bioenergetic state following graded traumatic brain injury in rats: determination by phosphorus 31 magnetic resonance spectroscopy. J Neurotrauma 1988;5:315-330
60. Dedeurwaerdere S, Callaghan PD, Pham T, et al. PET imaging of brain inflammation dury early epileptogenesis in a rat model of temporal lobe epilepsy. EJNMMI Res 2012;2:60.

61. Jones NC, Nguyen T, Corcoran NM, et al. Targeting hyperphosphorylated tau with sodium selenate suppresses seizures in rodent models. Neurobiol Dis 2012;45:897-901.

62. Virdee K, Cumming P, Caprioli D, et al. Applications of positron emission tomography in animal models of neurological and neuropsychiatric disorders. Neurosci Biobehav Rev 2012;36:1188-1216.

63. Chien DT, Bahri S, Szardenings AK, et al. Early clinical PET imaging results with the nivel PHF-tau radioligand [F-18]-T807. J Alzheimers Dis 2013;34:457-468.

64. Harhausen D, Sudmann V, Khojasteh U, et al. Specific imaging of inflammation with $18 \mathrm{dDa}$ translocator protein ligand DPA-714 in animal models of epilepsy and stroke. PLoS One $2013 ; 8:$ e69529.

65. Goffin K, Van Paesschen W, Dupont P, Van Laere K. Longitudinal microPET imaging of brain glucose metabolism in rat lithiumpilocarpine model of epilepsy. Exp Neurol 2009;217:205-209.

66. Guo Y, Gao F, Wang S, Ding Y, Zhang H, Wang J, Ding MP. In vivo mapping of temporospatial changes in glucose utilization in rat brain during epileptogenesis: an $18 \mathrm{~F}$-fluorodeoxyglucose-small animal positron emission tomography study. Neuroscience 2009;162: 972-979.

67. Blumenfeld H. Functional MRI studies of animal models in epilepsy. Epilepsia 2007;48:18-26.

68. Meyer-Lindenberg, A. From maps to mechanisms through neuroimaging of schizophrenia. Nature 2010;468:194-202.

69. Ogawa S, Lee TM, Kay AR, Tank DW. Brain magnetic resonance imaging with contrast dependent on blood oxygenation. Proc Natl Acad Sci U S A 1990; 87:9868-9872.

70. Ogawa S, Lee T. Blood oxygen level dependent MRI of the brain: effects of seizure induced by kainic acid in the rat. Proc Soc Magn Reson Med 1992;1:501.

71. Airaksinen AM, Niskanen JP, Chamberlain R, et al. Simultaneous fMRI and local field potential measurements during epileptic seizures in medetomidine-sedated rats using raser pulse sequence. Magn Reson Med 2010;64:1191-1199.

72. Airaksinen AM, Hekmatyar SK, Jerome N, et al. Simultaneous BOLD fMRI and local field potential measurements during kainic acid-induced seizures. Epilepsia 2012;53:1245-1253.

73. Mishra AM, Bai X, Purcaro MJ, et al. Interictal resting functional connectivity in Wag/Rij rats: a possible biomarker of epilepsy. Epilepsia 2009;50:363.

74. Mishra AM, Bai X, Motelow JA, et al. Increased resting functional connectivity in spike-wave epilepsy in WAG/Rij rats. Epilepsia 2013;54:1214-1222.

75. Nersesyan H, Hyder F, Rothman DL, Blumenfeld H. Dynamic fMRI and EEG recordings during spike-wave seizures and generalized tonic-clonic seizures in WAG/Rij rats. J Cereb Blood Flow Metab 2004;24:589-599.

76. Tenney JR, Duong TQ, King JA, Ludwig R, Ferris CF. Corticothalamlic modulation during absence seizures in rats: a functional MRI assessment. Epilepsia 2003;44:1133-1140.

77. Schmidt MH, Pohlmann-Eden B. Neuroimaging in epilepsy: the state of the art. Epilepsia 2011;52(Suppl. 4):49-51.

78. Galanopoulou AS, Moshe, S. In search of epilepsy biomarkers in the immature brain: goals, challenges and strategies. Biomark Med 2011;5:615-628.

79. Bernasconi A, Bernasconi N. Unveiling epileptogenic lesions: the contribution of image processing. Epilepsia 2011;52(Suppl. 4):20 24.

80. VanLandingham KE, Heinz ER, Cavazos JE, Lewis DV. Magnetic resonance imaging evidence of hippocampal injury after prolonged focal febrile convulsions. Ann Neurol 1998;43:413-426. 
81. Gomes WA, Shinnar, S. Prospects for imaging-related biomarkers of human epileptogenesis: a critical review. Biomark Med 2011;5: 599-606.

82. Natsume J, Bernasconi N, Miyauchi M, Naiki M, Yokotsuka T, Sofue A, Bernasconi A. Hippocampal volumes and diffusionweighted image findings in children with prolonged febrile seizures. Acta Neurol Scand 2007;115(4 Suppl.):25-28.

83. Bartolomei F, Regis J, Donnet A, Gastaut JL. Development of focal chronic epilepsy following focal status epilepticus in adult patients. Neurophysiol Clin 1999;29:271-276.

84. Provenzale JM, Barboriak DP, VanLandingham K, MacFall J, Delong D, Lewis DV. Hippocampal MRI signal hyperintensity after febrile status epilepticus is predictive of subsequent mesial temporal sclerosis. AJR Am J Roentgenol 2008;190:976-983.

85. Shinnar S, Bello JA, Chan S, et al. MRI abnormalities following febrile status epilepticus in children: the FEBSTAT study. Neurology 2012;79:871-877.

86. Nordli DR, Jr., Moshé SL, Shinnar S, et al. Acute EEG findings in children with febrile status epilepticus: results of the FEBSTAT study. Neurology 2012;79:2180-2186.

87. Angeleri F, Majkowski J, Cacchiò G, et al. Posttraumatic epilepsy risk factors: one-year prospective study after head injury. Epilepsia 1999; 40:1222-1230

88. Kumar R, Gupta RK, Husain M, et al. Magnetization transfer MR imaging in patients with posttraumatic epilepsy. AJNR Am J Neuroradiol 2003;24:218-224.

89. Messori A, Polonara G, Carle F, Gesuita R, Salvolini U. Predicting posttraumatic epilepsy with MRI: prospective longitudinal morphologic study in adults. Epilepsia 2005;46:1472-1481.

90. Cendes F. Neuroimaging predictors of AED resistance in new-onset epilepsies. Epilepsia 2011;52(Suppl. 4):7-9.

91. Freeman JL, Coleman LT, Wellard RM, et al. MR imaging and spectroscopic study of epileptogenic hypothalamic hamartomas: analysis of 72 cases. AJNR Am J Neuroradiol 2004;25:450-462.

92. Sisodiya SM. Wiring, dysmorphogenesis and epilepsy: a hypothesis. Seizure 1995;4:169-185.

93. Cepeda C, André VM, Levin MS, et al. Epileptogenesis in pediatric cortical dysplasia: the dysmature cerebral developmental hypothesis. Epilepsy Behav 2006;9:219-235.

94. Bernhardt BC, Worsley KJ, Kim H, Evans AC, Bernasconi A, Bernasconi N. Longitudinal and cross-sectional analysis of atrophy in pharmacoresistant temporal lobe epilepsy. Neurology 2009;72: $1747-1754$.

95. Gross DW. Diffusion tensor imaging in temporal lobe epilepsy. Epilepsia 2011;52(Suppl. 4):32-34.

96. Morita ME, Yasuda CL, Betting LE, et al. MRI and EEG as longterm seizure outcome predictors in familial mesial temporal lobe epilepsy. Neurology 2012;79:2349-2354.

97. Bernhardt BC, Rozen DA, Worsley KJ, Evans AC, Bernasconi N, Bernasconi A. Thalamo-cortical network pathology in idiopathic generalized epilepsy: insights from MRI-based morphometric correlation analysis. Neuroimage 2009;46:373-381.

98. Butler T, Zaborszky L, Wang X, et al. Septal nuclei enlargement in human temporal lobe epilepsy without mesial temporal sclerosis. Neurology 2013;80:487-491.

99. Cavus I, Pan JW, Hetherington HP, et al. Decreased hippocampal volume on MRI is associated with increased extracellular glutamate in epilepsy patients. Epilepsia 2008;49:1358-1366.

100. Gong G, Shi F, Concha L, Beaulieu C, Gross DW. Insights into the sequence of structural consequences of convulsive status epilepticus: a longitudinal MRI study. Epilepsia 2008;49:1941-1945.

101. Widjaja E, Blaser S, Miller E, et al. Evaluation of subcortical white matter and deep white matter tracts in malformations of cortical development. Epilepsia 2007;48:1460-1469.
102. Iannetti P, Papetti L, Nicita F, et al. Developmental anomalies of the medial septal area: possible implication for limbic epileptogenesis. Childs Nerv Syst 2011;27:765-770.

103. Pan JW, Williamson A, Cavus I, et al. Neurometabolism in human epilepsy. Epilepsia 2008;49(Suppl. 3):31-41.

104. Alkonyi B, Chugani HT, Juhász C. Transient focal cortical increase of interictal glucose metabolism in Sturge-Weber syndrome: implications for epileptogenesis. Epilepsia 2011;52:1265-1272.

105. Moosa AN, Gupta A, Jehi L, et al. Longitudinal seizure outcome and prognostic predictors after hemispherectomy in 170 children. Neurology 2013;80:253-260.

106. Juhasz C. The impact of positron emission tomography imaging on the clinical management of patients with epilepsy. Expert Rev Neurother 2012;12:719-732.

107. Szelies B, Sobesky J, Pawlik G, et al. Impaired benzodiazepine receptor binding in peri-lesional cortex of patients with symptomatic epilepsies studied by [(11)C]-flumazenil PET. Eur J Neurol 2002;9: 137-142.

108. Sata Y, Matsuda K, Mihara T, Aihara M, Yagi K, Yonekura Y. Quantitative analysis of benzodiazepine receptor in temporal lobe epilepsy: [(125)I]iomazenil autoradiographic study of surgically resected specimens. Epilepsia 2002;43:1039-1048.

109. Morimoto K, Tamagami H, Matsuda K. Central-type benzodiazepine receptors and epileptogenesis: basic mechanisms and clinical validity. Epilepsia 2005;46(Suppl. 5):184-188.

110. Mazzini L, Cossa FM, Angelino E, Campini R, Pastore I, Monaco F. Posttraumatic epilepsy: neuroradiologic and neuropsychological assessment of long-term outcome. Epilepsia 2003;44:569-574.

111. Bernasconi A, Bernasconi N, Natsume J, Antel SB, Andermann F, Arnold DL. Magnetic resonance spectroscopy and imaging of the thalamus in idiopathic generalized epilepsy. Brain 2003;126:24472454.

112. Park SA, Kim GS, Lee SK, et al. Interictal epileptiform discharges relate to $1 \mathrm{H}-\mathrm{MRS}$-detected metabolic abnormalities in mesial temporal lobe epilepsy. Epilepsia 2002;43:1385-1389.

113. Kuzniecky R, Hugg J, Hetherington $\mathrm{H}$, et al. Predictive value of $1 \mathrm{H}$ MRSI for outcome in temporal lobectomy. Neurology 1999;53: 694-698.

114. Pan JW, Spencer DD, Kuzniecky RR, Duckrow RB, Hetherington $\mathrm{H}$, Spencer SS. Metabolic networks in epilepsy by MR spectroscopic imaging. Acta Neurol Scand 2012;126:411-420.

115. Waites AB, Briellmann RS, Saling MM, Abbott DF, Jackson GD. Functional connectivity networks are disrupted in left temporal lobe epilepsy. Ann Neurol 2006;59:335-343.

116. Bettus G, Guedj E, Joyeux F, et al. Decreased basal fMRI functional connectivity in epileptogenic networks and contralateral compensatory mechanisms. Hum Brain Mapp 2009;30:1580-1591.

117. Pereira FR, Alessio A, Sercheli MS, et al. Asymmetrical hippocampal connectivity in mesial temporal lobe epilepsy: evidence from resting state fMRI. BMC Neurosci 2010;11:66.

118. Giblin KA, Blumenfeld H. Is epilepsy a preventable disorder? New evidence from animal models. Neuroscientist 2010;16:253-275.

119. Zheng P, Shultz SR, Hovens CM, Velakoulis D, Jones NC, O'Brien TJ. Hyperphosphorylated tau is implicated in acquired epilepsy and neuropsychiatric comorbidities. Mol Neurobiol 2013 Dec 10 [Epub ahead of print].

120. Francois J, Koning E, Ferrandon A, Nehlig A. The combination of topiramate and diazepam is partially neuroprotective in the hippocampus but not antiepileptogenic in the lithium-pilocarpine model of temporal lobe epilepsy. Epilepsy Res 2006;72:147-163.

121. Mani R, Pollard J, Dichter MA. Human clinical trials in antiepileptogenesis. Neurosci Lett 2011;497:251-256.

122. Loscher W, Klitgaard H, Twyman RE, Schmidt D. New avenues for anti-epileptic drug discovery and development. Nat Rev Drug Discov 2013;12:757-776. 